Revista de Metalurgia

Abril-Junio 2020, 56 (2), e168

ISSN-L: 0034-8570

https://doi.org/10.3989/revmetalm.168

\title{
NOTA TÉCNICA
}

\section{Proceso de reparación de carriles de tranvía de acero aleado con vanadio y su validación mediante modelado por elementos finitos (MEF)}

\author{
Daniel R. Galán-Rivera ${ }^{\mathrm{a}, \bigotimes}$, Manuel J. Orviz-Theodosius ${ }^{\mathrm{b}}$, Miguel Vigil ${ }^{\mathrm{b}, \mathrm{c}}$, \\ Daniel Miranda ${ }^{\mathrm{d}}$, Francisco J. Belzunce-Varela ${ }^{\mathrm{a}}$ \\ ${ }^{a}$ Universidad de Pyiedo. Departamęnto de Ciencia de los Materiales y Metalurgia,
C San Francisco 3, 33003 Oviedo, Asturias, Espana \\ 'Innvel2Consulting, C/Santiago, 2 - Bajo, 33210 Gijón, Asturias, España \\ 'Universidad de Oviedo, Departamento 33003 Gviedo, Asción y Prospección de Minas, C/ San Francisco 3, \\ ${ }^{\mathrm{d}}$ Centro Tecnológico IDONIAL, Parque Empresarial PEPA, Calafates, 33417, Avilés, Asturias \\ Autor para la correspondencia: galandanielramon@gmail.com
}

Enviado: 24 Octubre 2019; Aceptado: 12 Febrero 2020; Publicado on-Line: 28 Agosto 2020

\begin{abstract}
RESUMEN: La reparación in-situ de los carriles de tranvía mediante soldeo por arco eléctrico es un proceso ampliamente extendido que se utilizar para incrementar la vida útil en servicio de los carriles. Sin embargo, las nuevas resinas poliméricas empleadas en los embebidos de estas vías limitan las temperaturas que se pueden alcanzar en la reparación, ya que por encima de $170{ }^{\circ} \mathrm{C}$, la mayoría de estos polímeros se degradan. Por esta razón, es necesario desarrollar un proceso de soldeo rápido, seguro y económicamente viable, que al mismo tiempo garantice que los carriles no superan dicha temperatura en la superficie de contacto citada. En este estudio, se realizó la reparación de un acero de carril R290V (aleado con vanadio) mediante la deposición de un metal de aporte inoxidable mayoritariamente austenítico a través de un proceso de soldeo por arco eléctrico e hilo tubular (FCAW). El procedimiento de soldeo aplicado se ha diseñado y validado mediante simulación numérica por elementos finitos, habiéndose demostrado la posibilidad de realizar el recargue "in situ" del carril de tranvía sin que en la región del embebido se superen $140{ }^{\circ} \mathrm{C}$ y sin que se forme martensita en ningún punto de la Zona Afectada Térmicamente (ZAT).
\end{abstract}

PALABRAS CLAVE: Acero con vanadio; Carril; Desgaste; MEF; Recargue FCAW; Tranvía

Citar como/Citation: Galán-Rivera, D.R.; Orviz-Theodosius, M.J.; Vigil, M.; Miranda, D.; Belzunce-Varela, F.J. (2020). "Proceso de reparación de carriles de tranvía de acero aleado con vanadio y su validación mediante modelado por elementos finitos (MEF)". Rev. Metal. 56(2): e168. https://doi.org/10.3989/revmetalm.168

\begin{abstract}
Welding repair process of vanadium steel grooved rails and its validation by means of finite element modelization (FEM). Arc welding repair procedures are widely used to increase the life service of grooved rails on trams. However, the new polymeric embedments used with these rails limit the temperature that can be achieved during the repair process, as these materials degrade when they are heated above $170{ }^{\circ} \mathrm{C}$. To avoid this problem, an optimum weld repair procedure must be developed to ensure an economically and fast weld without exceeding the limited temperature of the embedment. In this study, the repair of the new R290V rail steel (vanadium alloyed) was carried out with an austenitic stainless-steel consumable and a flux core arc welding technology (FCAW). The procedure was designed and validated using a finite element simulation and it was proved that it is possible to repair these rails without even attaining above $140{ }^{\circ} \mathrm{C}$ in the embedment and without the formation of martensite.
\end{abstract}

KEYWORDS: FCA weld; FEM; Rail; Tramway; Vanadium steel; Wear

ORCID ID: Daniel R. Galán-Rivera (https://orcid.org/0000-0001-6810-0110); Manuel J. Orviz-Theodosius (https:// orcid.org/0000-0002-1282-7166); Miguel Vigil (https://orcid.org/0000-0003-3687-6867); Daniel Miranda (https://orcid. org/0000-0001-6225-7491); Francisco J. Belzunce-Varela (https://orcid.org/0000-0002-3049-2654)

Copyright: (C) 2020 CSIC. Este es un artículo de acceso abierto distribuido bajo los términos de la licencia de uso y distribución Creative Commons Reconocimiento 4.0 Internacional (CC BY 4.0). 


\section{INTRODUCION}

El crecimiento de la población mundial y su masificación en las grandes ciudades exige el desarrollo de unos transportes públicos eficientes que permitan desplazar al mayor número de personas con el menor impacto medioambiental posible. Para el año 2050 , se estima que el $70 \%$ de la población mundial se concentrará en grandes ciudades (Cho, 2018), por lo que ya resulta fácil prever los problemas relacionados con la contaminación, la habitabilidad y la movilidad en estas megapoblaciones (Borck, 2019). Es entonces necesario el desarrollo de "medios de transporte verdes", que reduzcan las emisiones de contaminantes, sustituyendo a los medios de transporte convencionales (Goldman y Gorham, 2006). Si bien el mercado privado de los medios de transporte eléctricos es aún demasiado pequeño, las instituciones públicas deben apostar por el desarrollo de estas tecnologías (Carrese et al., 2014). Aquí es donde entra en juego el uso del tranvía cuya elevada capacidad de movimiento de pasajeros y sus bajos niveles contaminantes, lo posicionan como un medio de transporte alternativo eficiente del máximo interés (Martín et al., 2014). Además, como los carriles que se utilizan para esta aplicación van embebidos en la calzada, el tranvía es un medio de transporte compatible con el resto de los medios de transporte públicos que existen actualmente en las ciudades.

Con el desarrollo de los tranvías, es necesario mejorar en paralelo las propiedades de los carriles que constituyen la vía (Pacyna, 2008; Carrol et al., 2013). Al aumentar las cargas por eje y las velocidades, también se incrementan los esfuerzos y solicitaciones que debe soportar la vía, especialmente en las regiones en curva. En el caso de los carriles de tranvía, estas solicitaciones pueden incluso duplicarse ya que, por la disposición de la vía en las calzadas de las ciudades, los radios de las curvas pueden llegar a ser inferiores a 20 m (Sandor y Ramsey, 2012). Además, como estos carriles se encuentran normalmente embebidos en la calzada en moldes de polímero, resulta muy compleja su sustitución cuando han sufrido un desgaste excesivo. Por esta razón, resulta más económica la reparación de los carriles desgastados que su sustitución por carriles nuevos (TRCP, 2012). Una de las soluciones más extendidas para la reparación de los carriles es el recargue por soldadura con arco eléctrico, que permite la reparación de los carriles desgastados mediante la deposición de un acero con la misma o con diferente composición química y propiedades que las del carril original (Marqueteeken et al., 2008; Zahiri et al., 2014). Además, en estos casos, el aporte térmico aplicado debe ser menor que el utilizado normalmente con objeto de evitar el deterioro térmico del embebido plástico y para evitar este problema es conveniente llevar a cabo simulaciones mediante análisis previos por elementos finitos (FEA) del proceso de reparación (Rosenthal, 1946; Wetsby, 1968; Rykalin et al., 1971; Goldack et al., 1984; Bullon et al., 2007; Martínez et al., 2009), para garantizar temperaturas máximas en el embebido inferiores al límite preestablecido, evitar pruebas innecesarias y optimizar, de este modo, los costes y el tiempo empleados en la reparación.

Con la incorporación de los nuevos aceros de carril aleados con vanadio, no solo es posible incrementar la vida útil de los carriles frente al desgaste, sino que también se mejora su soldabilidad, ya que permiten mantener los mismos niveles de dureza en la superficie de rodadura utilizando composiciones químicas con un menor porcentaje de carbono (ArcelorMittal, 2018; Solano-Alvarez et al., 2019). De cualquier manera, resulta necesario desarrollar procedimientos de soldeo que permitan la reparación "in situ" de estos carriles, utilizando una temperatura de precalentamiento lo suficientemente baja como para evitar el deterioro del embebido plástico que soporta la vía.

\section{METODO EXPERIMENTAL}

\subsection{Materiales y procedimiento de soldeo}

En este estudio se utilizó el nuevo grado de acero de carril de tranvía aleado con vanadio desarrollado por la compañía ArcelorMittal y cuya denominación comercial es R290V. Siguiendo las recomendaciones de trabajos previos (Sandor et al., 2013), se seleccionó como metal de aporte un acero inoxidable de matriz austenítica con una pequeña fracción de martensita $(15 \mathrm{CrMn})$. La aleación con cromo le confiere su carácter inoxidable y la adición de manganeso estabiliza la austenita como fase matriz mayoritaria, hecho que incrementa sus propiedades anti-desgaste, bajo la condición de desgaste por rodadura, en virtud de la transformación de la microestructura austenítica en martensita que tiene lugar bajo las cargas normales de servicio del carril (efecto TRIP) (Vuorinen et al., 2009).

Las composiciones químicas de los metales base (R290V) y de aporte (15CrMn) se recogen en la Tabla 1. La composición química del acero base se ha obtenido mediante análisis gravimétrico en el caso del silicio, utilizando un analizador Leco CS230 en el caso del carbono, y mediante espectroscopía atómica (AES) para el resto de los elementos (Solano-Alvarez et al., 2019). La composición del metal de aporte fue suministrada por el fabricante.

Se utilizó la geometría del carril 54 G1 (41 GPU) de acuerdo a la normativa UNE-EN 14811 (2011). Para simular el efecto del daño debido al desgaste en los carriles situados en una curva, se realizó un mecanizado de $20 \mathrm{~mm}$ de profundidad en la esquina de la cabeza de uno de los carriles (Fig. 1). Tras el mecanizado citado, el perfil fue recargado utilizando la tecnología de soldeo por arco eléctrico e hilo tubular (FCAW). 
TABLA 1. Composición química del acero base y del aporte (\% en peso)

\begin{tabular}{|c|c|c|c|c|c|c|c|c|}
\hline Metal & Matriz & $\mathbf{C}$ & $\mathbf{S i}$ & Mn & $\mathrm{Cr}$ & $\mathbf{N i}$ & Mo & $\mathbf{V}$ \\
\hline $15 \mathrm{CrMn}$ & Austeno-Martensítica & $0,3-0,4$ & $0,5-0,7$ & $13,5-13,8$ & $15,0-16,0$ & $1,6-1,8$ & $0,7-0,8$ & $0,6-0,7$ \\
\hline R290V & Perlítica & $0,5-0,6$ & $0,3-0,5$ & $0,9-1,1$ & $<0,15$ & - & - & $0,1-0,2$ \\
\hline
\end{tabular}

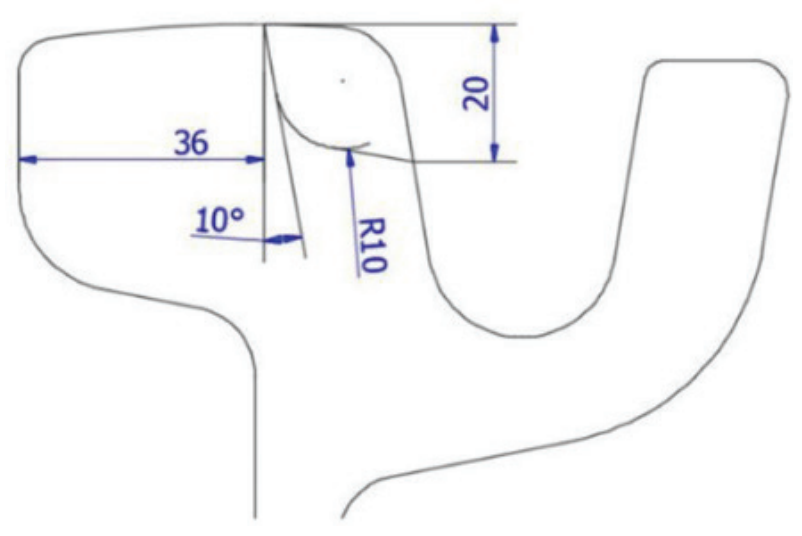

Figura 1. Diseño del mecanizado utilizado para simular el daño debido al desgaste en curva del carril de tranvía 54G1.

En la ejecución de esta reparación "in situ” es muy importante destacar la limitación que existe en cuanto a la temperatura máxima que se permite alcanzar en el embebido plástico que rodea al carril, que normalmente debe fijarse en $150-170^{\circ} \mathrm{C}$, ya que en caso contrario el embebido se degradaría desprendiendo además unos humos tóxicos muy contaminantes (Fig. 2) (Sandor y Ramsey, 2012).

\subsection{Caracterización térmica, metalúrgica y de durezas llevada a cabo después del recargue}

En la reparación se llevó a cabo el control de la temperatura en la superficie del recargue y en la superficie de éste en contacto con el embebido. Con este fin, en primer lugar, se taladraron 6 agujeros de $4 \mathrm{~mm}$ de diámetro y $15 \mathrm{~mm}$ de profundidad en uno de los laterales de la cabeza del carril (Fig. 3). El primer agujero se taladró a $50 \mathrm{~mm}$ de uno de los extremos del carril, mientras que el resto de los agujeros se taladraron sucesivamente cada $190 \mathrm{~mm}$. En el interior de estos agujeros, se instalaron termopares de contacto, fijados mediante una pasta sellante, con objeto de medir la temperatura de la región próxima a la superficie recargada. Además, en los puntos intermedios entre cada agujero se colocaron en contacto con la superficie del carril otros termopares soldados con objeto de medir la temperatura de la superficie en contacto con el embebido (Fig. 4).

Para el precalentamiento de la muestra se utilizó un equipo de precalentamiento con llama alimentado mediante propano. Se estableció una temperatura de precalentamiento de $100{ }^{\circ} \mathrm{C}$ en la zona

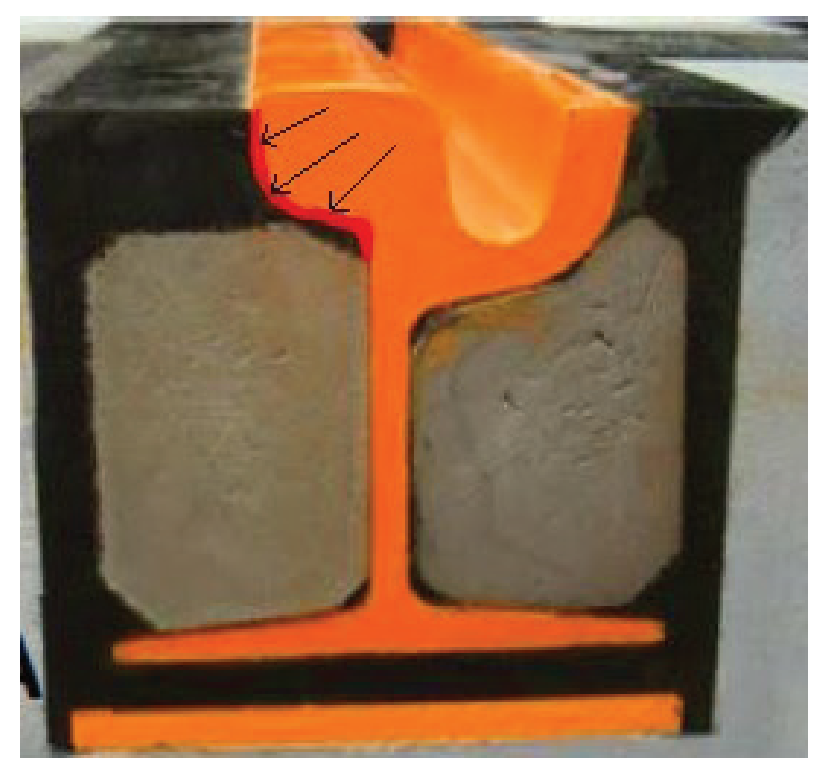

FIgURA 2. Ejemplo de la región sensible a la temperatura de un embebido polimérico en un carril de tranvía.

próxima a la superficie de soldeo, lo que suponía un calentamiento de la zona en contacto con el embebido de en torno a $60{ }^{\circ} \mathrm{C}$.

Una vez realizado el proceso experimental de recargue del carril, se extrajeron dos lajas transversales, la primera de una sección donde se había situado uno de los agujeros que contenía un termopar de contacto (Fig. 5a), y la segunda de una región en la que se había dispuesto un termopar soldado (Fig. 5b). Sobre la primera de estas secciones se llevó a cabo la caracterización metalográfica y se realizaron cadenas de dureza. La muestra se fresó y desbastó con lijas de carburo de silicio hasta una granulometría de P120, para finalmente realizar una macrografía de su superficie. Para revelar el recargue de acero inoxidable se utilizó un ataque electrolítico basado en una disolución acuosa de $\mathrm{NaOH}$ al $20 \%$, mientras que la macroestructura del acero base y de sus zonas afectadas térmicamente se revelaron con Nital al 15\%.

Para la evaluación de la dureza se realizaron dos cadenas localizadas a $1 \mathrm{~mm}$ de la superficie del carril en las regiones de inicio y fin del recargue (líneas LI y LII indicadas en la Fig. 6). Se utilizó el método Vickers con una carga de $10 \mathrm{~kg}$ (HV10), siguiendo los criterios de la norma UNE-EN 6507-1 (2018). Se diferenció entre la zona afectada térmicamente debida a los cordones de la primera capa (ZAT inferior, LII) y la zona afectada térmicamente debida 
a los últimos cordones depositados (ZAT superior, LI), ya que éstas son las zonas más críticas

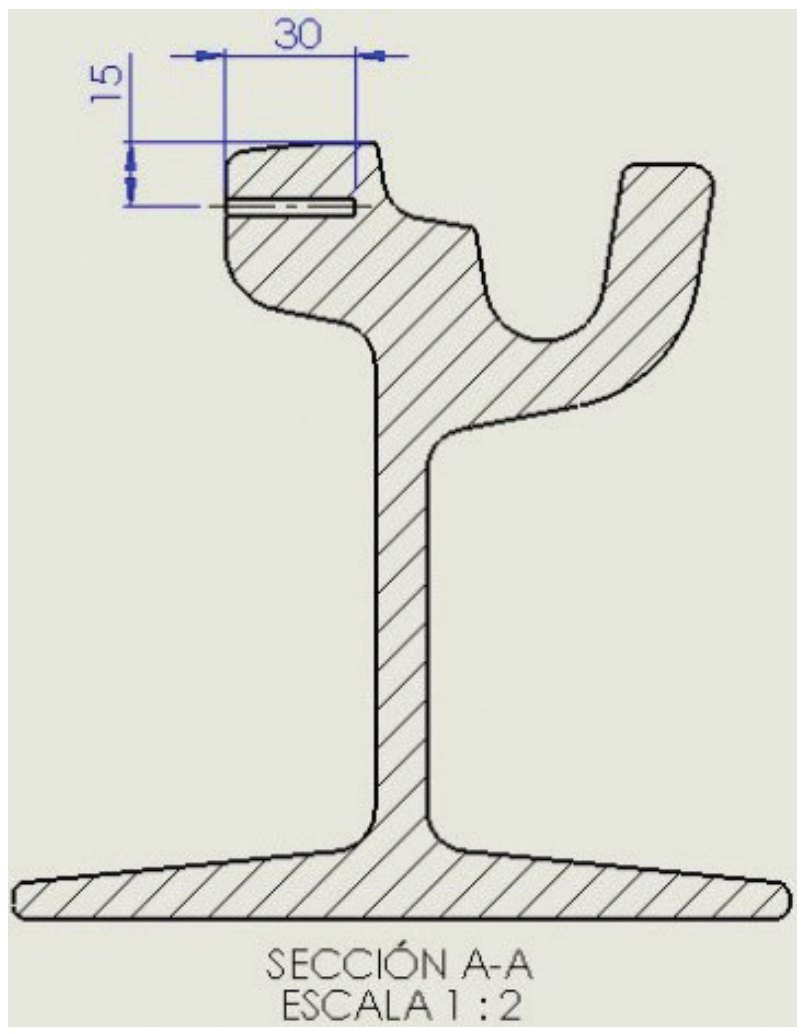

FIgURA 3. Distribución de los agujeros en donde se situaron los termopares de contacto. del recargue. Hay que destacar que, en el proceso industrial de reparación del carril de tranvía, tras el recargue se debe realizar un reperfilado de la región depositada a fin de recuperar la geometría inicial que tenía el carril.

\subsection{Simulación del proceso de recargue por soldeo}

Para la simulación numérica del proceso de recargue se utilizó un software de elementos finitos SYSWELD. Se realizaron varias simulaciones en 2 y 3 dimensiones utilizando diferentes parámetros de soldeo y temperaturas de precalentamiento.

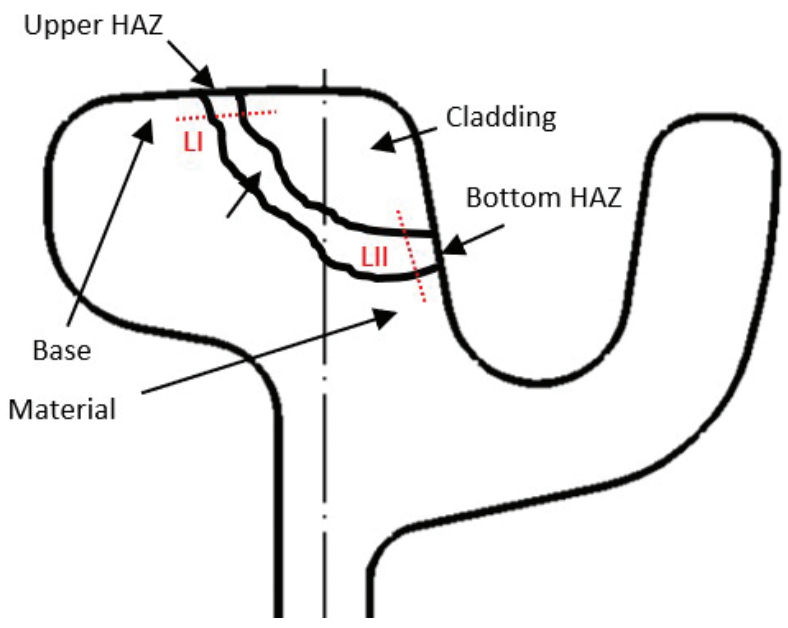

Figura 6. Disposición de los perfiles de dureza (LI y LII) realizados sobre el carril recargado y reperfilado.

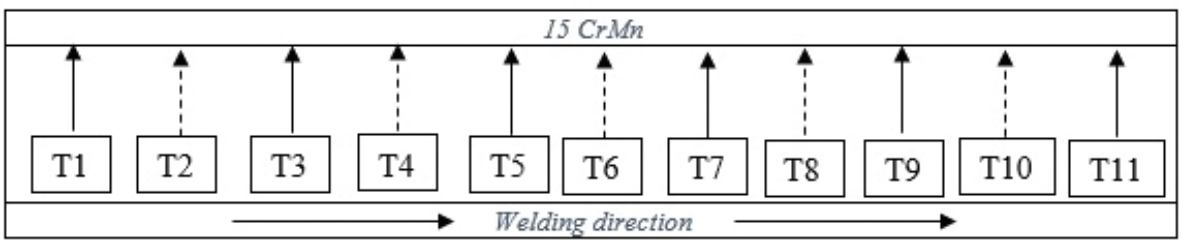

Figura 4. Disposición de los termopares en el carril recargado. En línea continua se sitúa la localización de los termopares de contacto y en línea discontinua la de los termopares soldados.
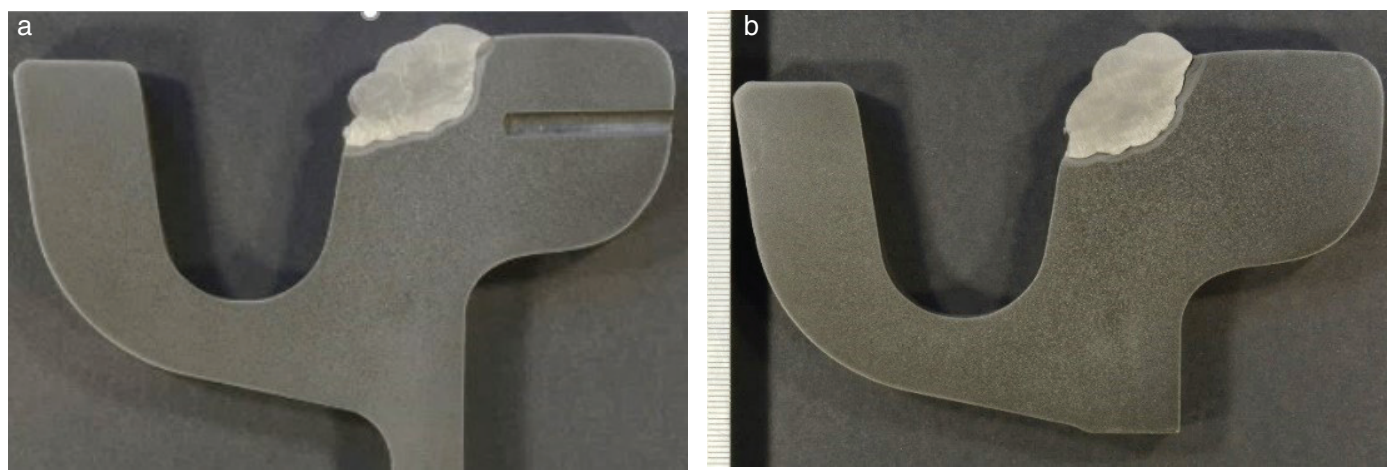

Figura 5. Macrografías extraídas de la región en donde se situaba (a) un termopar de contacto y (b) un termopar soldado. 
Se utilizó un modelo de fuente de calor basado en dos elipsoides combinados (Goldack et al., 1984), de manera que la evolución del calor Q en el frente se puede calcular a partir de la expresión Ec. (1):

$Q(x, y, z, t)=\frac{\sqrt[6]{3} f_{f} q}{a b c \pi \sqrt{\pi}} e^{-3 x^{2} / a^{2}} e^{-3 y^{2} / b^{2}} e^{-3[z+v+(\tau-t)]^{2} / c^{2}}$

mientras que, en la parte posterior al paso de la fuente de calor, éste se expresa mediante la Ec. (2):

$Q(x, y, z, t)=\frac{\sqrt[6]{3} f_{r} q}{a b c \pi \sqrt{\pi}} e^{-3 x^{2} / a^{2}} e^{-3 y^{2} / b^{2}} e^{-3[x+v+(\tau-t)]^{2} / c^{2}}$

siendo $\mathrm{a}, \mathrm{b}$ y c las dimensiones de los semiejes del elipsoide, $v$ la desviación típica, $\tau$ el factor de retardo necesario para definir la posición de la fuente para un tiempo t igual a $0, \mathrm{y} \mathrm{f}_{\mathrm{r}} \mathrm{y}$ $\mathrm{f}_{\mathrm{f}}$ unos factores que fraccionan el calor en el frente y tras el elipsoide y cuya suma es igual a 2. A partir de las macrografías expuestas en la Fig. 5, se han obtenido los parámetros dimensionales del elipsoide, ancho y profundidad de la zona fundida, al tiempo que se ha tomado la distancia delante de la fuente de calor igual al ancho y la distancia tras la fuente el doble del ancho (Goldack et al., 1984).

Por otro lado, las propiedades físicas del acero constitutivo del carril R290V (conductividad térmica, calor específico y coeficiente de dilatación térmica), variables con la temperatura, que se han utilizado en estos cálculos, se han obtenido con la ayuda del software de simulación JMatPro.

\subsubsection{Simulaciones iniciales}

Definidos unos parámetros de soldeo iniciales extraídos de la bibliografía (Sandor y Ramsey, 2012), y que se muestran en la Tabla 2, se realizaron varias simulaciones preliminares, sin aplicar precalentamiento alguno, en las que se depositaron 14 cordones formando 7 capas, más una final de revenido, para completar la geometría del perfil de un carril de 4 metros de longitud (Fig. 7). En todas las simulaciones se ha utilizado el mismo mallado compuesto principalmente por elementos cuadriláteros (en 2 y en 3 dimensiones) con integración lineal de los nodos y un refinamiento de la malla en la zona de interés (zona del recargue y ZAT del mismo) (Fig. 7). En las diferentes evaluaciones se fue incrementando el tiempo de espera entre pasadas necesario para evitar que la superficie del carril en contacto con el embebido superase $170{ }^{\circ} \mathrm{C}$ (puntos marcados con flechas en la Fig. 7).

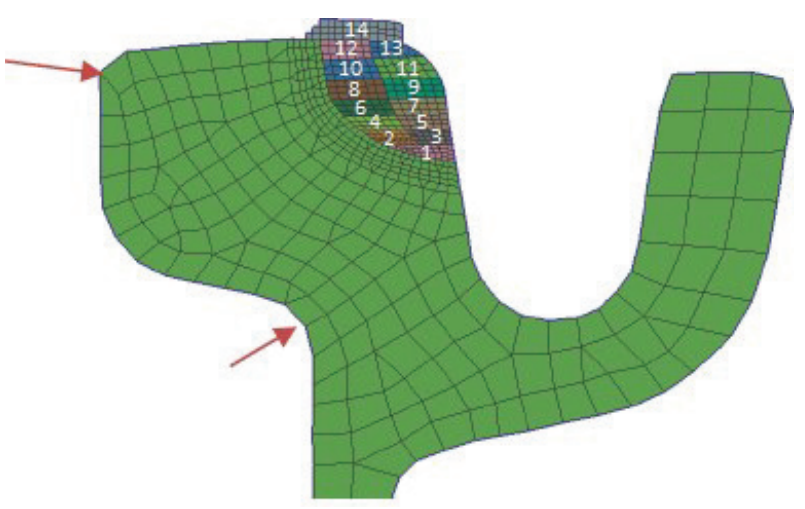

FIGURA 7. Mallado utilizado en las simulaciones numéricas iniciales de la ZAT del recargue. Las flechas indican los puntos de medida sobre los que se controló que la temperatura no superase $170{ }^{\circ} \mathrm{C}$.

TABLA 2. Parámetros de soldeo utilizados en las simulaciones iniciales

\begin{tabular}{|c|c|c|c|c|}
\hline Cordón & Velocidad, $\mathrm{cm} \cdot \mathrm{min}^{-1}$ & Voltaje, V & Intensidad, A & Aporte térmico, $\mathbf{J} \cdot \mathrm{mm}^{-1}$ \\
\hline 1 & 90 & $31-32$ & $240-250$ & 510 \\
\hline $\begin{array}{l}2 \\
3\end{array}$ & 86 & $31-32$ & $240-250$ & 540 \\
\hline $\begin{array}{l}4 \\
5\end{array}$ & 83 & $31-32$ & $240-250$ & 560 \\
\hline $\begin{array}{l}6 \\
7\end{array}$ & 76 & $31-32$ & $240-250$ & 610 \\
\hline $\begin{array}{l}8 \\
9\end{array}$ & 70 & $31-32$ & $240-250$ & 660 \\
\hline $\begin{array}{l}10 \\
11\end{array}$ & 65 & $31-32$ & $240-250$ & 710 \\
\hline $\begin{array}{l}12 \\
13\end{array}$ & 60 & $31-32$ & $240-250$ & 770 \\
\hline 14 & 45 & $31.5-32$ & $250-260$ & 1040 \\
\hline
\end{tabular}




\subsubsection{Simulaciones finales}

Tras las simulaciones preliminares se puso de manifiesto que, con los parámetros de soldeo teóricos utilizados en las simulaciones anteriores, no era posible llenar completamente la junta, ya que el rendimiento del proceso era inferior a uno, en virtud de las pérdidas térmicas siempre existentes en la realidad, por lo que se tuvo que incrementar el número de cordones, para depositar 20 cordones en 7 capas, incluyendo el cordón final de revenido (Fig. 8) y, además, se utilizó también una temperatura de

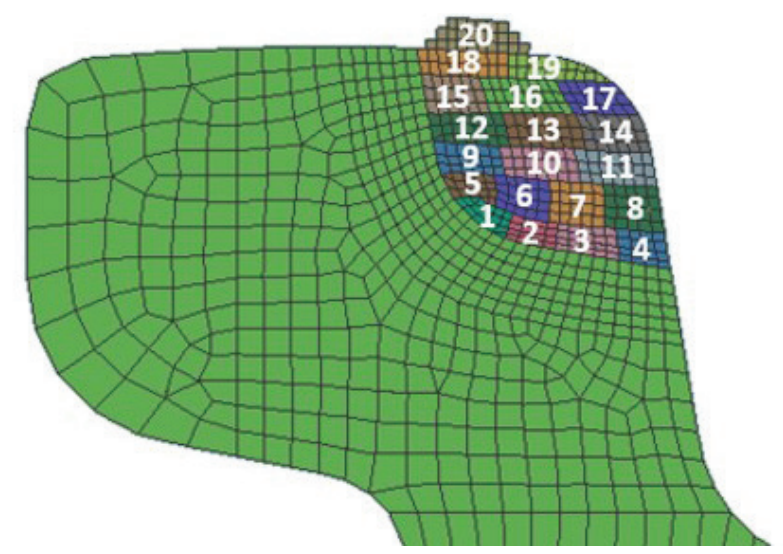

FIgURA 8. Distribución de los cordones de recargue en la simulación final de la reparación. precalentamiento de $60{ }^{\circ} \mathrm{C}$, con objeto de asegurar que no se formaba martensita en la ZAT. En este caso, para adecuar las simulaciones a la realidad, el carril simulado tenía 1 metro de longitud.

Los parámetros de soldeo utilizados (número de cordones, velocidad de avance, voltaje, intensidad y aporte térmico) tanto en las pruebas experimentales como en estas simulaciones (ESAB, 2019) se recogen en la Tabla 3. Los cordones se han numerado siguiendo la secuencia establecida en la Fig. 8, comenzando el recargue por la parte inferior izquierda, continuando de izquierda a derecha y de abajo a arriba.

En estas simulaciones se midió también la evolución de la temperatura durante el proceso completo de recargue en los mismos nodos en los que se habían colocado los termopares (Fig. 4 y Fig. 7).

\section{RESULTADOS}

\subsection{Simulaciones numéricas}

\subsubsection{Simulaciones iniciales}

Se comenzó utilizando un tiempo de espera entre cordones de 60 segundos, con los parámetros de soldeo que se habían indicado en la Tabla 2. Bajo esta condición se pudieron depositar, sin superar la temperatura máxima especificada en la superficie, solo 5 cordones en el recargue realizado sin precalentar.

TABLA 3. Parámetros de soldeo utilizados en las simulaciones finales

\begin{tabular}{|c|c|c|c|c|c|}
\hline & Cordón* & $\mathrm{V}_{\text {soldeo }}, \mathrm{cm} \cdot \mathrm{min}^{-1}$ & Intensidad, A & Voltaje, V & Aporte Térmico, $\mathbf{J} \cdot \mathrm{mm}^{-1}$ \\
\hline \multirow[t]{4}{*}{$1^{\mathrm{a}}$ capa } & 1 & 70 & $115-130$ & $31,2-31,6$ & $262-300$ \\
\hline & 2 & 60 & $123-167$ & $29,1-30,2$ & $304-428$ \\
\hline & 3 & & & & \\
\hline & 4 & & & & \\
\hline \multirow[t]{4}{*}{$2^{\mathrm{a}}$ capa } & 5 & 60 & $145-183$ & $28,6-29,9$ & $353-465$ \\
\hline & 6 & & & & \\
\hline & 7 & & & & \\
\hline & 8 & & & & \\
\hline \multirow[t]{3}{*}{$3^{\mathrm{a}}$ capa } & 9 & 55 & $190-213$ & $28,7-29,4$ & $506-581$ \\
\hline & 10 & & & & \\
\hline & 11 & & & & \\
\hline \multirow[t]{3}{*}{$4^{\mathrm{a}}$ capa } & 12 & 50 & $177-202$ & $29,2-29,8$ & $527-558$ \\
\hline & 13 & & & & \\
\hline & 14 & & & & \\
\hline \multirow[t]{3}{*}{$5^{\text {a capa }}$} & 15 & 45 & $160-185$ & $29,6-30,4$ & $537-637$ \\
\hline & 16 & 55 & $170-200$ & $29,5-30,3$ & $465-562$ \\
\hline & 17 & & & & \\
\hline \multirow[t]{2}{*}{$6^{\mathrm{a}}$ capa } & 18 & 40 & $180-210$ & $29,1-29,8$ & $668-798$ \\
\hline & 19 & & & & \\
\hline $7^{\mathrm{a}}$ capa & 20 & 40 & $170-220$ & $28,9-30,4$ & $627-853$ \\
\hline
\end{tabular}

*véase la Fig. 8 

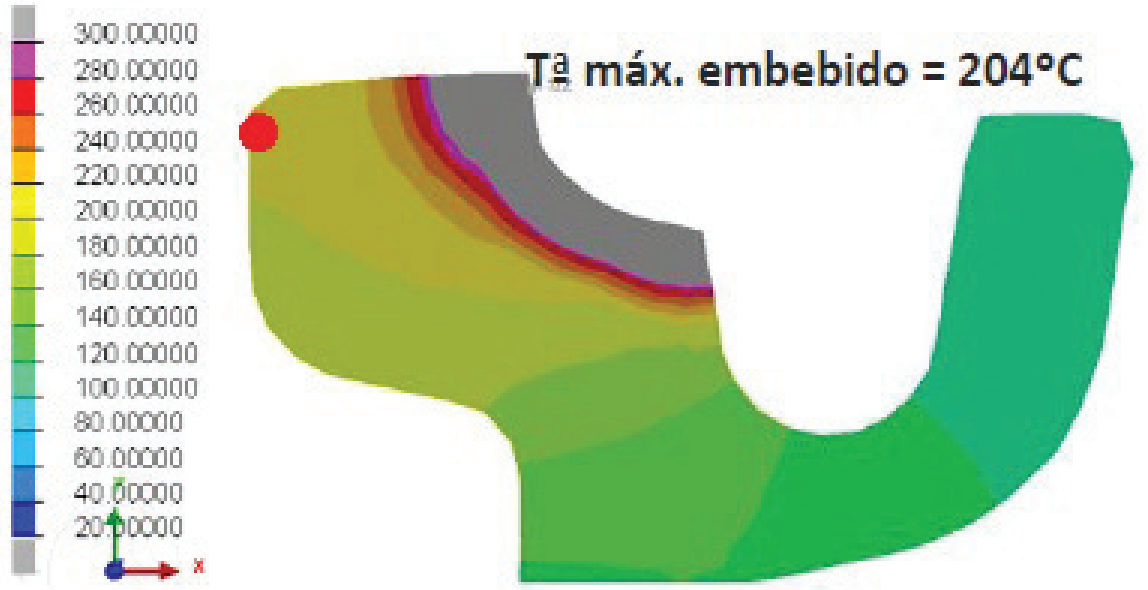

FIGURA 9. Distribución de las temperaturas máximas alcanzadas en el carril con la deposición de 14 cordones, sin precalentar $\left(20^{\circ} \mathrm{C}\right)$, con una espera de $300 \mathrm{~s}$ entre cada cordón. El círculo rojo indica la región en dónde se midió la máxima temperatura en la zona de contacto con el embebido.

TABLA 4. Secuencia de tiempos optimizada para el recargue de un carril de 4 metros

\begin{tabular}{lcccc}
\hline $\mathbf{N}^{\mathbf{0}}$ Cordón & $\mathbf{t}_{\mathbf{0}}(\mathbf{m i n})$ & $\mathbf{t}_{\mathbf{w}}(\mathbf{m i n})$ & $\mathbf{t}_{\mathbf{n p}}(\mathbf{m i n})$ & \\
\hline 1 & 0 & 4,5 & 0,5 \\
2 & 5 & 4,6 & 0,5 \\
3 & 10,1 & 4,6 & 0,5 & \\
4 & 15,2 & 4,8 & 0,5 & \\
5 & 20,5 & 4,8 & 0,5 & \\
6 & 25,8 & 5,2 & 0,5 & \\
7 & 31,6 & 5,2 & 0,5 & \\
8 & 37,4 & 5,7 & 0,5 & \\
9 & 43,6 & 5,7 & 4,3 & \\
10 & 53,6 & 6,2 & 0,5 & \\
11 & 60,3 & 6,2 & 4 & \\
12 & 70,2 & 6,7 & 0,5 & \\
13 & 77,4 & 6,7 & 3 & \\
14 & 87,4 & 8,9 & - & $\mathrm{t}_{\text {total }}=96$ min \\
\hline
\end{tabular}

Se fue incrementando así el tiempo entre pasadas a 180,240 y finalmente a $300 \mathrm{~s}$ sin conseguir evitar el calentamiento excesivo en la región de la superficie de la cabeza en contacto con el embebido (Fig. 9), por lo que, para superar esta limitación, se decidió realizar esperas superiores en determinadas pasadas concretas (cordones finales). Se diseñó así una secuencia de pasadas óptima (Tabla 4), con la que se logró mantener la temperatura máxima de la superficie del carril por debajo del rango preestablecido de $150-170{ }^{\circ} \mathrm{C}$. En esta tabla se indica el tiempo, en minutos, para el comienzo de cada pasada $\left(\mathrm{t}_{0}\right)$, el tiempo que tarda en realizarse cada pasada de 4 metros de longitud $\left(t_{w}\right)$ y el tiempo de espera que se deja entre pasada y pasada para que enfrié el

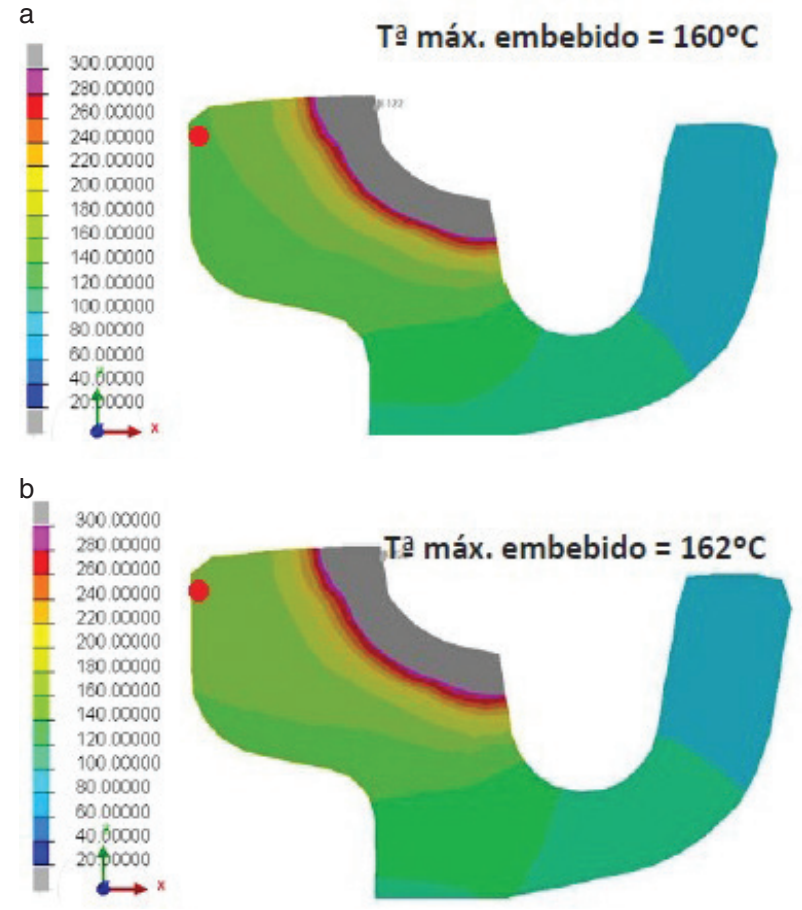

FIGURA 10. Distribución de la temperatura en el carril tras depositar 14 cordones con los tiempos de espera definidos en la Tabla 4: a) sin precalentar $\left(20^{\circ} \mathrm{C}\right)$ y b) utilizando un precalentamiento de $50{ }^{\circ} \mathrm{C}$.

carril (tnp). Como se puede ver en la misma tabla, el tiempo total $\left(\mathrm{t}_{\text {total }}\right)$ para la realización completa del recargue de un carril de 4 metros de longitud sería de 96 minutos.

Con la secuencia de pasadas optimizada definida, se consiguió que la temperatura máxima en la superficie de la cabeza del carril al completar el proceso de recargue no superase $160{ }^{\circ} \mathrm{C}$ (Fig. 10a). Además, 
TABLa 5. Tiempo inicial $\left(\mathrm{t}_{\mathrm{o}}\right)$, tiempo de soldeo $\left(\mathrm{t}_{\mathrm{w}}\right)$ y tiempo entre pasadas $\left(\mathrm{t}_{\mathrm{i}}\right)$ medidos experimentalmente en cada pasada. Tiempo total del proceso completo de recargue de $1 \mathrm{~m}$ de carril $\left(\mathrm{t}_{\text {total }}\right)$.

\begin{tabular}{|c|c|c|c|c|c|c|c|c|c|c|}
\hline 15CrMn & Cordón 1 & Cordón 2 & Cordón 3 & Cordón 4 & Cordón 5 & Cordón 6 & Cordón 7 & Cordón 8 & Cordón 9 & Cordón 10 \\
\hline$t_{o}(s)$ & 2055 & 2457 & 2855 & 3079 & 3662 & 4504 & 4932 & 5173 & 5463 & 6605 \\
\hline $\mathrm{t}_{\mathrm{w}}(\mathrm{s})$ & 92 & 155 & 104 & 117 & 107 & 131 & 108 & 105 & 122 & 122 \\
\hline \multirow[t]{2}{*}{$\mathrm{t}_{\mathrm{i}}(\mathrm{s})$} & 310 & 243 & 120 & 466 & 735 & 297 & 133 & 185 & 1020 & 148 \\
\hline & Cordón 11 & Cordón 12 & Cordón 13 & Cordón 14 & Cordón 15 & Cordón 16 & Cordón 17 & Cordón 18 & Cordón 19 & Cordón 20 \\
\hline$t_{o}(s)$ & 6875 & 7291 & 7798 & 8247 & 8711 & 9624 & 10247 & 10907 & 11482 & 12287 \\
\hline $\mathrm{t}_{\mathrm{w}}(\mathrm{s})$ & 120 & 120 & 148 & 140 & 143 & 160 & 111 & 175 & 175 & 175 \\
\hline$t_{i}(s)$ & 296 & 387 & 301 & 324 & 770 & 463 & 549 & 400 & 630 & - \\
\hline
\end{tabular}

utilizando estos mismos tiempos, se realizaron unas nuevas simulaciones, aplicando ahora un cierto precalentamiento y se comprobó que con una temperatura de precalentamiento de $50^{\circ} \mathrm{C}$, apenas se registró un incremento de 2 grados en la temperatura de la superficie del carril en contacto con el embebido al final del proceso de recargue (Fig. 10b).

\subsubsection{Simulaciones finales}

En la Tabla 5 se muestran ahora los tiempos en los que se comenzó la deposición de cada cordón $\left(\mathrm{t}_{\mathrm{o}}\right)$, el tiempo empleado en el recargue del mismo $\left(t_{w}\right)$, y el tiempo de espera que hubo de utilizarse al finalizar el depósito de cada cordón $\left(\mathrm{t}_{\mathrm{i}}\right)$, con objeto de mantener la región crítica del embebido plástico por debajo de $150{ }^{\circ} \mathrm{C}$, utilizando ahora 7 capas y 20 pasadas y los parámetros de soldeo indicados en la Tabla 3. En la misma tabla, se indica también el tiempo total necesario para completar el proceso de recargue de un metro de carril.

Para la validación de las simulaciones, se compararon los ciclos térmicos medidos experimentalmente con los diferentes termopares con los ciclos térmicos calculados numéricamente en los nodos correspondientes.

A modo de ejemplo, en la Fig. 11 se ha representado la evolución de la temperatura en uno de los termopares soldados, que mide la temperatura del carril en la zona de contacto con el embebido (termopar T4, Fig. 4), junto a la predicción numérica realizada en esa misma localización. Cada uno de los 20 picos corresponde a la deposición de cada cordón. Se puede observar una buena correspondencia entre las medidas experimentales y las obtenidas mediante simulación numérica, si bien, en el caso de la simulación, el precalentamiento es instantáneo mientras que en la prueba experimental se requiere un tiempo de casi 1000 s para alcanzar la temperatura de precalentamiento de $60^{\circ} \mathrm{C}$. En la Tabla 6 se han representado las temperaturas máximas de cada uno de los picos, así como el error relativo entre las medidas experimentales y las numéricas, que se situó en un error medio del $12.8 \%$. Se pone de manifiesto también que, de acuerdo con las condiciones preestablecidas, en ningún momento se ha superado una temperatura de $140{ }^{\circ} \mathrm{C}$ en el embebido.

\subsection{Análisis microestructural y perfiles de dureza}

Tal y como se puede observar en la Fig. 12, la máxima dureza medida en la ZAT se situó en torno a $350 \mathrm{HV}$, en la zona de la ZAT inferior, y en torno a $400 \mathrm{HV}$ en la región de la ZAT superior. Esta diferencia de dureza se justifica en función del mayor revenido de la ZAT que ha tenido lugar en las primeras pasadas con respecto a la ZAT de la última pasada, que fue únicamente revenida por efecto de la pasada de sobrerevenido final, depositada tras completar la geometría del recargue. La dureza del metal de aporte se sitúa en torno a un valor medio de $280 \mathrm{HV}$, que es ligeramente inferior al valor de dureza del metal base (300-320 HV). Además, se observa una diferencia de dureza que se sitúa en torno a $20 \mathrm{HV}$ (320 frente a $300 \mathrm{HV}$ ) entre las zonas del acero base cercanas a la ZAT superior e inferior del proceso de recargue. Esta heterogeneidad en la dureza se justifica en virtud de la acumulación de calor durante el enfriamiento final tras la laminación en caliente del carril y es, por lo tanto, independiente del proceso de recargue. En virtud de la geometría de la cabeza del carril, la disipación de calor en su zona inferior es menor y la menor velocidad de enfriamiento de esta región tras la laminación es el origen del leve ablandamiento detectado en esta zona.

Sobre las mismas muestras en las que se realizaron las medidas de dureza, se realizó también la caracterización microestructural. La microestructura de la ZAT inferior está compuesta principalmente por perlita, con un tamaño de grano muy fino, G13 (Fig. 13a). Esta microestructura es fruto de un enfriamiento relativamente rápido que, sin embargo, no fue suficiente para la formación de martensita, en virtud de la temperatura de precalentamiento utilizada y mantenida durante todo el soldeo. En la zona de la ZAT superior se ha obtenido una 

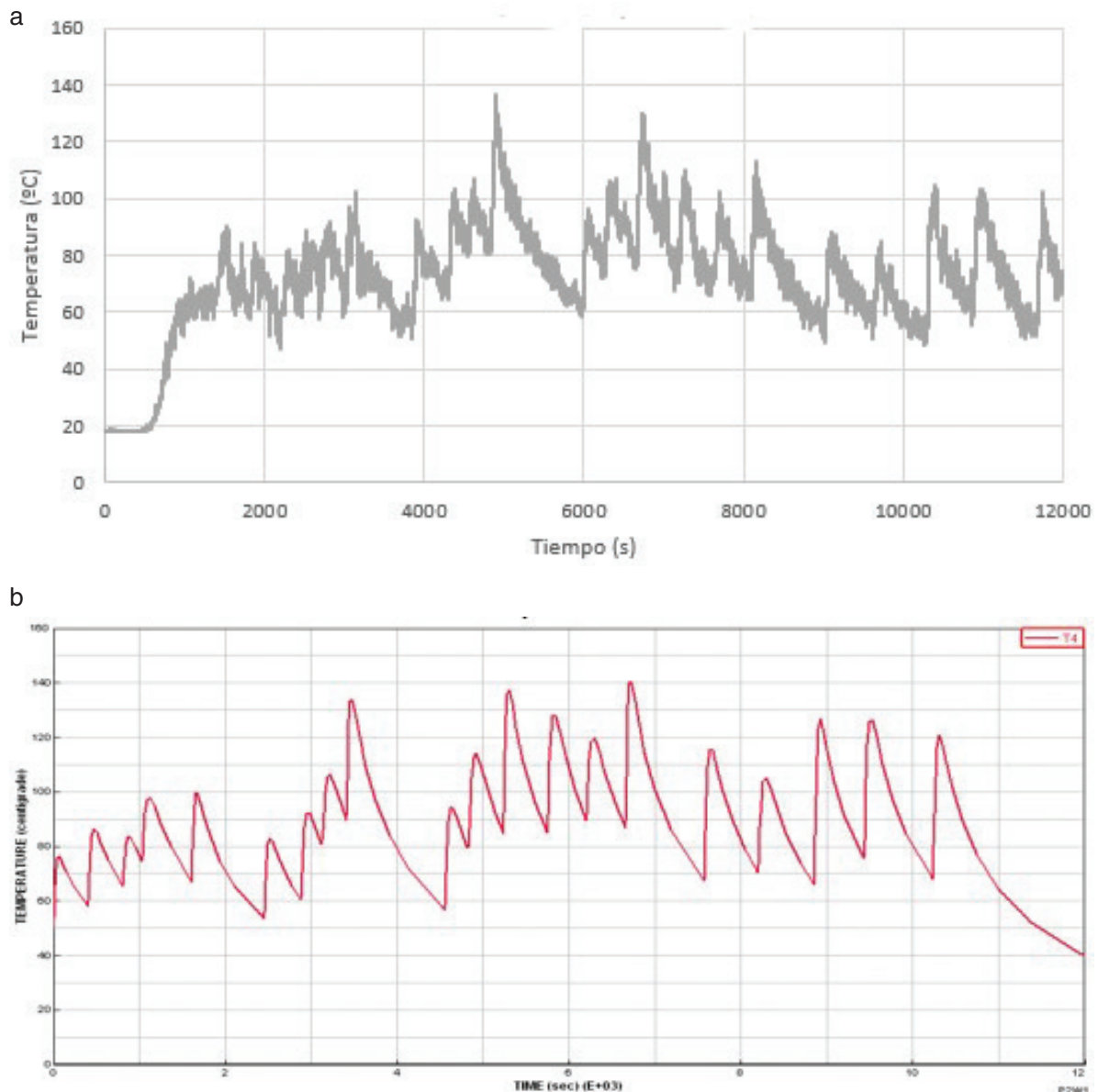

Figura 11. Ejemplo de la evolución de la temperatura en función del tiempo medido en el termopar T4 soldado a la superficie de contacto con el embebido (a) y su correspondiente simulación numérica (b).

TABLA 6. Máximas temperaturas medidas en la superficie del carril en contacto con el embebido en las pruebas experimentales y en las simulaciones numéricas (Termopar T4, véase Fig. 4)

\begin{tabular}{lccccccc}
\hline & $\begin{array}{c}\text { Experimental } \\
{ }^{\mathbf{0}} \mathbf{C}\end{array}$ & $\begin{array}{c}\text { Simulación } \\
{ }^{\mathbf{0}} \mathbf{C}\end{array}$ & $\begin{array}{c}\text { Error } \\
\text { Relativo } \%\end{array}$ & & $\begin{array}{c}\text { Experimental } \\
{ }^{\mathbf{}} \mathbf{C}\end{array}$ & $\begin{array}{c}\text { Simulación } \\
{ }^{\mathbf{0}} \mathbf{C}\end{array}$ & $\begin{array}{c}\text { Error } \\
\text { Relativo \% }\end{array}$ \\
\hline Cordón 1 & 89 & 78 & 12,4 & Cordón 11 & 104 & 112 & 7,7 \\
Cordón 2 & 81 & 87 & 7,4 & Cordón 12 & 119 & 128 & 7,6 \\
Cordón 3 & 87 & 84 & 3,4 & Cordón 13 & 107 & 119 & 11,2 \\
Cordón 4 & 88 & 98 & 11,4 & Cordón 14 & 100 & 120 & 20,0 \\
Cordón 5 & 103 & 100 & 2,9 & Cordón 15 & 112 & 140 & 25,0 \\
Cordón 6 & 88 & 82 & 6,8 & Cordón 16 & 87 & 116 & 33,3 \\
Cordón 7 & 101 & 91 & 9,9 & Cordón 17 & 84 & 105 & 25,0 \\
Cordón 8 & 103 & 107 & 3,9 & Cordón 18 & 104 & 128 & 23,1 \\
Cordón 9 & 135 & 133 & 1,5 & Cordón 19 & 102 & 127 & 24,5 \\
Cordón 10 & 94 & 94 & 0,0 & Cordón 20 & 102 & 121 & 18,6 \\
\hline
\end{tabular}

microestructura mayoritariamente perlítica, aunque con una muy pequeña fracción de bainita y con ferrita contorneando un borde de grano austenítico ya más grosero, G8, (Fig. 13b). En esta zona se observa el efecto de la pasada de sobrerevenido que, sumada a la acumulación de calor generada durante todo el proceso, justifica el mayor tamaño de grano medido en esta región. Además, las trazas de bainita que 
10 • D.R. Galán-Rivera et al.

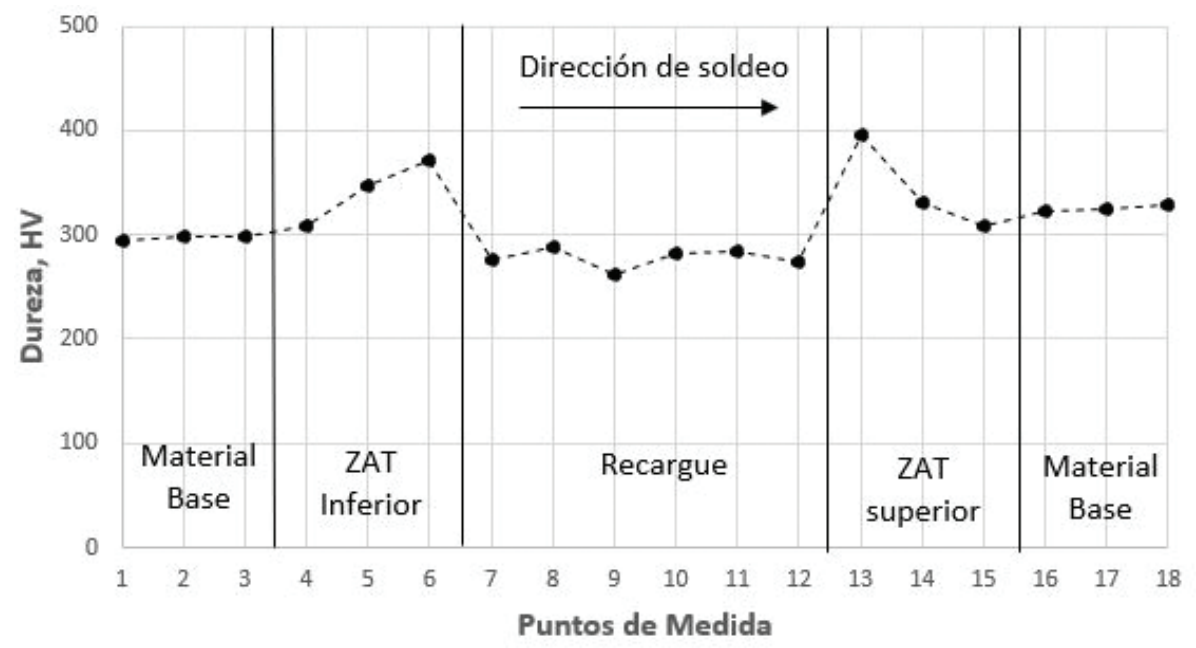

FIgURA 12. Perfiles de dureza medidos en las localizaciones LI (ZAT superior) y LII (ZAT inferior), de acuerdo con el esquema de la Fig. 6.
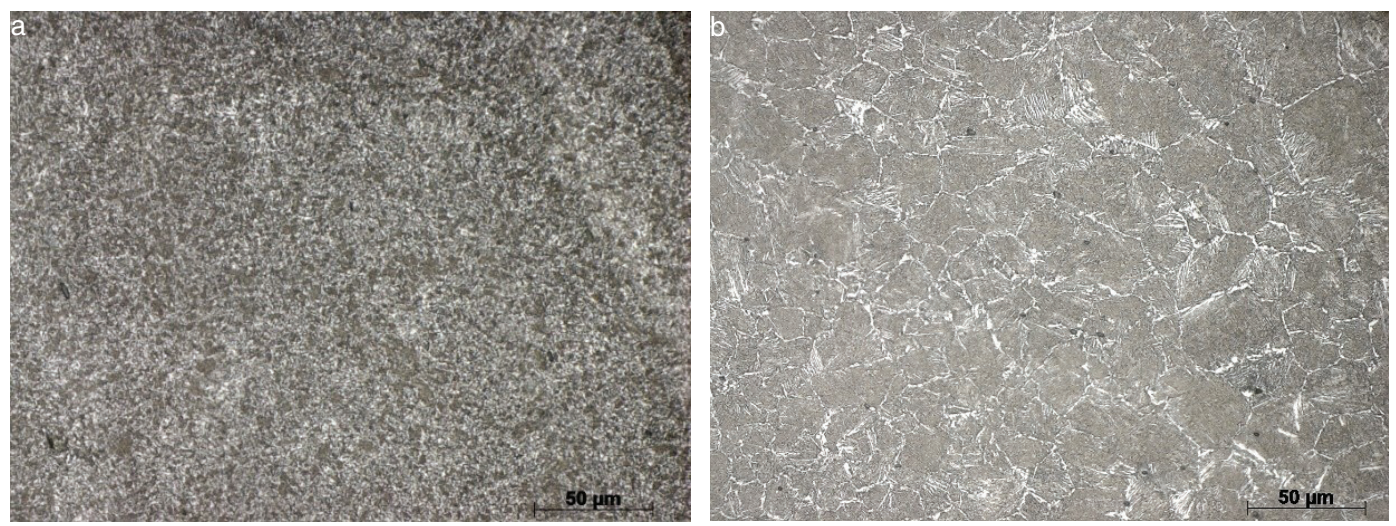

FIgURA 13. Micrografía de la ZAT inferior (a) y de la ZAT superior (b), 500x.
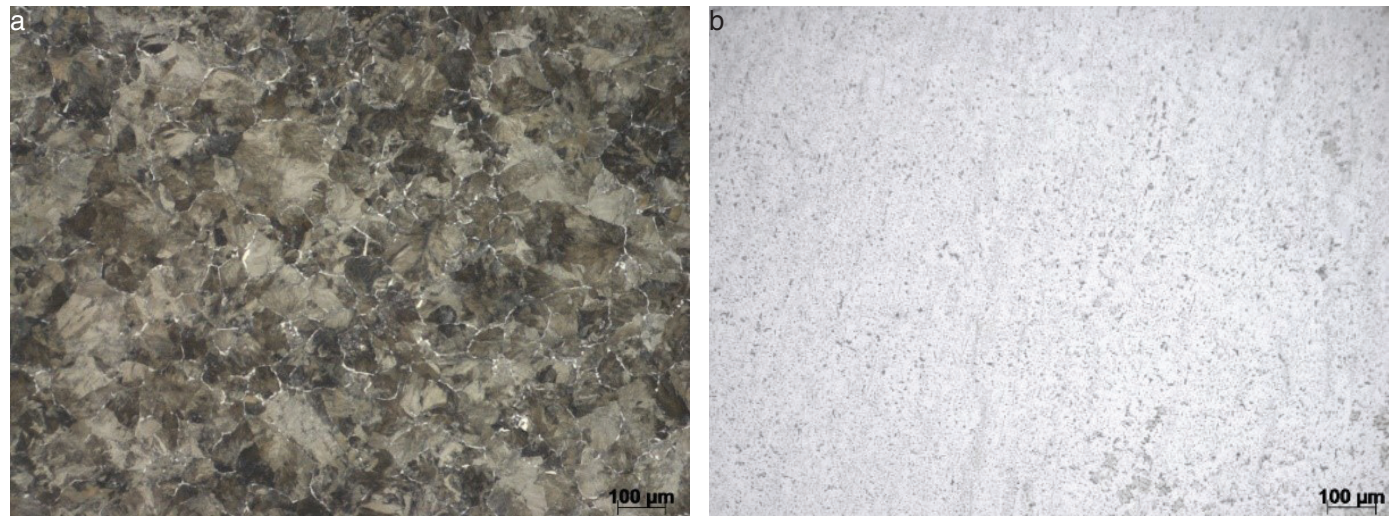

Figura 14. Micrografía del acero base (a) y del metal de aporte (b), 500x. 
aparecen entre la microestructura ferrito-perlítica justifican la mayor dureza medida en esta región. Por último, en la Fig. 14. se presenta tanto la micrografía realizada sobre el metal base, en la que se aprecia la microestructura ferrito-perlítica del acero R290V, como la micrografía realizada sobre el metal de aporte, que es mayoritariamente austenítica, con algunas trazas de martensita.

\section{CONCLUSIONES}

Las principales aportaciones del trabajo realizado se pueden resumir en las siguientes conclusiones:

- Se ha demostrado la posibilidad de realizar la reparación "in situ" de un acero de carril de tranvía R290V desgastado, mediante la deposición de un metal de aporte mayoritariamente austenítico, con un proceso de soldeo por arco eléctrico e hilo tubular utilizando una temperatura de precalentamiento inferior a $100^{\circ} \mathrm{C}$ y sin alcanzar una temperatura superior a $150{ }^{\circ} \mathrm{C}$ en el embebido plástico que rodea la cabeza del carril.

- Las durezas obtenidas en las ZATs del acero base presentan una dureza inferior a $400 \mathrm{HV}$, no observándose microestructuras martensíticas. A este respecto, resulta de vital importancia el adecuado diseño de la secuencia de pasadas que garantice una velocidad de enfriamiento adecuada para evitar microestructuras frágiles y el uso de una pasada final de sobrerevenido que se eliminará en una operación de mecanizado final.

- Se ha logrado una buena correlación entre las medidas experimentales de los ciclos térmicos y las obtenidas mediante las simulaciones numéricas. De este modo la simulación numérica del proceso de soldeo permite predecir de modo fiable las temperaturas que se alcanzan en la región del embebido y ajustar la secuencia de pasadas o los parámetros de soldeo con objeto de evitar temperaturas excesivas en esta región.

\section{AGRADECIMIENTOS}

Esta investigación ha sido financiada con la ayuda a empresas para la realización de Doctorados Industriales: "Oviedo Siembra Talento", destinadas a la contratación de estudiantes de Programa de Doctorado impartidos en la Universidad de Oviedo, financiados por el ayuntamiento de Oviedo. El autor agradece y aprecia la contribución de Josef Tec de la empresa ESI GROUP por sus consejos e indicaciones sobre el SYSWELD y a Felipe Álvarez de la fundación IDONIAL por la ayuda prestada en la caracterización experimental.

\section{REFERENCIAS}

ArcelorMittal (2018). Grooved Rails for Tramways. Technical Manual. ArcelorMittal Europe Long Products. Rails \& Special Sections. https://rails.arcelormittal.com/ documentos/12-english/download.

Borck, R. (2019). Public transport and urban pollution. Reg. Sci. Urban. Econ. 77, 356-366. https://doi.org/10.1016/j. regsciurbeco.2019.06.005.

Bullon, W., Acosta, J., Franco, R., Valverde, Q. (2007). Simulación de un proceso de soldadura mediante un modelo Termo-Mecánico considerando el efecto de esfuerzos residuales utilizando el Método de los Elementos Finitos. $8^{\circ}$ Congreso Iberoamericano de Ingeniería Mecánica. http://congreso.pucp.edu.pe/cibim8/pdf/20/20-38.pdf.

Carrese, S., Gemma, A., La Spada, S. (2014). An emission model to compare bus and tramway transport. Procedia Soc. Behav. Sci. 111, 1025-1034. https://doi.org/10.1016/j. sbspro.2014.01.137.

Carrol, R., Smith, H.M., Jaiswal, S. (2013). Rail steel with an excellent combination of wear properties and rolling contact fatigue resistance. U.S. Patent No 8430976.

Cho, S. (2018). 30 years working together solve Shangai's most pressing water problems. Worldbank. https://blogs.worldbank.org/water/30-years-working-together-solve-shanghai-s-most-pressing-water-problems.

ESAB (2019). Proceso de Soldadura FCAW - Alambre Tubular Relleno de Fundente. https://www.esab.com.ar/ar/sp/ education/blog/proceso-soldadura-fcaw-alambre-tubularrelleno-de-fundente-definiciones-del-proceso.cfm.

Goldack, J., Chakravarti, A., Bibby, M. (1984). A new finite element model for welding heat sources. Metall. Trans. B 15 (2), 299-305. https://doi.org/10.1007/BF02667333.

Goldaman, T., Gorham, R. (2006). Sustainable urban transport: Four innovative directions. Technol. Soc. 28 (1-2), 261-273. https://doi.org/10.1016/j.techsoc.2005.10.007.

Marqueteeken, A., Van Leuven, A., Kopf, F. (2008). Cost effective track maintenance, renewal \& refurbishment methods. Preventive maintenance of embedded tram tracks Rail wear in curves and special trackwork for trams. URBAN TRACK Project.

Martín, L., Calvo, F., Hermoso, A., De Oña, J. (2014). Analysis of light rail systems in Spain according to their type of funding. Procedia Soc. Behav. Sci. 162, 419-428. https:// doi.org/10.1016/j.sbspro.2014.12.223.

Martínez, E., Estrems, M., Miguel, V., Garrido, A., Guillén, J. A. (2009). Estado del arte de la modelización numérica de los procesos de soldadura por arco eléctrico empleados en construcción metálica. XIII Congreso Internacional de Ingeniería de Proyectos, Badajoz, pp. 8-10. https://www.aeipro.com/files/congresos/2009badajoz/ ciip09_0460_0475.2486.pdf.

Pacyna, J. (2008). The microstructure and properties of the new bainitic rail steels. J. Achiev. Mater. Manuf. Eng. 28 (1), 19-22.

Rosenthal, D. (1946). The theory of moving sources of heat and its application of metal treatments. ASME, Vol. 68, pp. 849-866.

Rykalin, N.N., Nikolaev, A.V., Goronkov, O.A (1971). Calculation of current density in anode spot of arc. Teplofiz. Vys. Temp. (9), 981-985.

Sandor, T., Ramsey, J. (2012). Microstructure development in embedded tram rails due to ESAB cyclic surface welding technology without preheating. ESAB Global Market.

Sandor, T., Ramsey, J., Dumovic, M., Wiseman, R. (2013). Onsite repair welding of rails. Metals New Zealand.

Solano-Alvarez, W., Fernandez Gonzalez, L., Bhadeshia, H.K.D.H. (2019). The effect of vanadium on the wear resistance of pearlitic grooved rails. Wear 436-437, 203004. https://doi.org/10.1016/j.wear.2019.203004.

TCRP (2012). Track design handbook for light rail transit. Second Edition, Report 155. Transportation Research Board, EEUU. https://doi.org/10.17226/22800.

UNE-EN 14811 (2011). Aplicaciones ferroviarias. Carriles para fines especiales: construcción asociada y acanalada, AENOR. https://www.une.org/encuentra-tu-norma/ busca-tu-norma/norma?c=N0046749. 
UNE-EN 6507-1 (2018). Metallic Materials. Vickers hardness test, AENOR.

Vuorinen, E., Wang, L., Stanojevic, S., Prakash, B. (2009). Influence of retained austenite on rolling-sliding wear resistance of austempered silicon alloyed steel. International Conference on Hot Sheet Metal Forming of High-Performance Steel, pp. 339-347.
Westby, O. (1968). Temperature Distribution in the Workpiece by Welding. Dept. of Metallurgy and Metals Working, The Technical Univ. of Norway. PhD Dissertation.

Zahiri, R., Sundaramoorthy, R., Lysz, P., Subramanian, C. (2014). Hardfacing using ferro-alloy powder mixtures by submerged arc welding. Surf. Coat. Tech. 260, 220-229. https://doi.org/10.1016/j.surfcoat.2014.08.076. 